\title{
Surgical outcome of extended liver resections for colorectal liver metastasis compared with standard liver resections
}

\author{
GO SHINKE $^{1}$, TAKEHIRO NODA ${ }^{1}$, HIDETOSHI EGUCHI ${ }^{1}$, YOSHIFUMI IWAGAMI ${ }^{1}$, \\ DAISAKU YAMADA ${ }^{1}$, TADAFUMI ASAOKA ${ }^{1}$, KOICHI KAWAMOTO ${ }^{1}$, KUNIHITO GOTOH $^{1}$, \\ SHOGO KOBAYASHI $^{1}$, YUTAKA TAKEDA ${ }^{1,2}$, MASAHIRO TANEMURA $^{1,3}$, TSUNEKAZU MIZUSHIMA ${ }^{1}$, \\ KOJI UMESHITA $^{4}$, YUICHIRO DOKI ${ }^{1}$ and MASAKI MORI ${ }^{1}$

\begin{abstract}
${ }^{1}$ Department of Gastroenterological Surgery, Graduate School of Medicine, Osaka University, Suita, Osaka 565-0871;
${ }^{2}$ Department of Surgery, Kansai Rosai Hospital, Amagasaki, Hyogo 660-8511; ${ }^{3}$ Department of Surgery, Osaka Police Hospital, Osaka 543-0035; ${ }^{4}$ Division of Health Science, Graduate School of Medicine, Osaka University, Suita, Osaka 565-0871, Japan
\end{abstract}

Received December 2, 2017; Accepted May 15, 2018

DOI: $10.3892 / \mathrm{mco} .2018 .1632$

\begin{abstract}
Colorectal liver metastatic lesions sometimes invade adjacent organs. A hepatectomy is often extended to include the involved adjacent organ to achieve negative surgical margins. The purpose of the present retrospective study was to evaluate the surgical outcomes of extended liver resections and patients' prognoses. The medical records of 178 patients with colorectal liver metastasis who underwent liver resections in the Department of Gastroenterological Surgery at Osaka University Hospital (Suita, Japan), from 2000 to 2015 were reviewed. These patients were divided into two groups: the extended resection group $(n=20)$ and the non-extended resection group $(\mathrm{n}=158)$. The disease-free and overall survival curves were estimated with the Kaplan-Meier method and analyzed with the log rank test. It was observed that the extended resection group had longer operation times and increased blood loss, however perioperative morbidity was similar. The organs resected most frequently were the diaphragm $(n=10)$ and inferior vena cava $(n=5)$. Overall survival rates in the extended resection group were lower compared with the non-extended resection group (5-year survival rates; 45.0 vs. $67.9 \%$ ), however the difference was not significant. It was indicated that the aggressive hepatectomy combined with resection of adjacent organs was an acceptable treatment with low perioperative morbidity. The overall survival rate may not be inferior to that of simple hepatectomy.
\end{abstract}

Correspondence to: Dr Hidetoshi Eguchi, Department of Gastroenterological Surgery, Graduate School of Medicine, Osaka University, 2-2 Yamadaoka, Suita, Osaka 565-0871, Japan E-mail: heguchi@gesurg.med.osaka-u.ac.jp

Key words: hepatectomy, diaphragm, inferior vena cava, colon cancer, liver cancer

\section{Introduction}

The incidence of colorectal cancer has increased 4-fold over the last 25 years in Japan. A recent study showed that $25 \%$ of patients with colorectal cancer had distant disease at diagnosis. The most common site of distant metastases was liver. During the natural course of colorectal carcinoma, liver metastasis develops in about half of the patients, and metastatic liver tumors are responsible for death in about two-thirds of those patients (1). For patients with untreated liver metastasis, the median survival is reported to be around 8 months (2). Recently, impressive development in systemic chemotherapy has improved the clinical response and survival rates of patients with colorectal liver metastasis. Nevertheless, it remains shorter than survival of patients who received curative resections. Therefore, when hepatic metastatic lesions are diagnosed as resectable, hepatectomy is the first treatment modality $(3,4)$. Although the rate of cure with the initial hepatic resection is $<25 \%$ (5), the 5-year survival rate of patients treated with complete resections of liver metastases was reported to be $40-50 \%(6,7)$.

For patients with colorectal liver metastasis involving adjacent organs, hepatectomy combined with a resection of the involved adjacent organ is selected to achieve negative surgical margins. Adjacent organs involved with metastatic liver tumors are mainly the diaphragm and inferior vena cava (IVC). Previously, a liver resection with a simultaneous diaphragm excision was associated with a greater incidence of perioperative morbidity and a significantly worse longterm outcome than liver resection alone $(8,9)$. In addition, the only treatment modality for curing a primary or metastatic liver tumor that invaded IVC was an aggressive hepatectomy combined with resection and reconstruction of IVC, which is a challenging treatment with high morbidity and mortality rates $(10,11)$.

Recently, surgical techniques have been developed $(12,13)$, and recent advances in combination chemotherapy have improved patients' survival. To our knowledge, few previous reports have studied short and long-term outcomes of patients who received a hepatectomy combined with resection of an 
adjacent organ for treating colorectal liver metastasis. In this study, we compared patients' characteristics between two groups: the extended liver resection group, which included patients that received a hepatectomy combined with resection of adjacent organs, and the non-extended resection group, which included patients that received a simple hepatectomy. We also evaluated the surgical outcomes and prognosis of patients that received extended liver resections.

\section{Patients and methods}

Patients. Between February 2000 and November 2015, 190 patients with a diagnosis of colorectal liver metastasis were treated with a liver resection in the Department of Gastroenterological Surgery at Osaka University Hospital. Written informed consent to receive perioperative management and surgery was obtained from all patients. The inclusion criteria were follows; the patients of colorectal liver metastasis who received a liver resection and elective surgery. The exclusion criteria were as follows: emergency or urgent surgery; viable metastatic lesion in the remnant liver after the resection and missing details in the medical records. Patients who met at least one of these exclusion criteria were excluded. The patients underwent routine pre-operative imaging studies, including enhanced chest and abdomen computed tomography $(\mathrm{CT})$, superparamagnetic iron oxide magnetic resonance imaging (MRI), and angiography. More recently, patients have been assessed with multiphasic dynamic CT, with ethoxybenzyl MRI, and 18F-Fluorodeoxyglucose positron emission tomography (FDG-PET), without angiography. Out of 190 patients with colorectal liver metastasis, twelve patients were excluded for further analysis; of these, seven patients had viable metastatic lesions in the remnant liver after the liver resection, and five patients had missing details in the medical records. Consequently, 178 eligible patients were included in the final analysis.

The 178 patients were divided into two groups: the extended resection group $(n=20)$ and the non-extended resection group $(n=158)$. We defined a non-extended resection as a hepatectomy alone, and an extended resection as a liver resection combined with a resection of adjacent organs that were directly invaded by the metastatic lesions of the liver. These two groups were analyzed and compared for clinical features, including sex, age, body weight, number of resections, timing of metastasis (synchronous or metachronous), tumor number, neoadjuvant therapy, distribution of hepatic lobes, Child-Pugh score, tumor size, stage of primary colorectal carcinoma (based on the International Union Against Cancer, 7th edition), adjuvant chemotherapy, usage of molecular targeted drugs in perioperative chemotherapy and the conversion cases from the unresectable liver metastasis to the resectable liver metastasis by intense systemic chemotherapy. The perioperative chemotherapy was defined as neoadjuvant chemotherapy and adjuvant chemotherapy in this study. Two groups were also compared for surgical outcomes, including operation time, blood loss, blood transfusion volume, resected liver weight, postoperative complications, histology, and postoperative hospital stay. The surgical curability of the resection was categorized as follows: R0 indicated that all gross disease was removed and the margins were histologically free of disease; R1 indicated that all gross disease was removed but the margins were histologically positive for disease; and R2 indicated that some residual gross disease remained after the resection (14). Complications were graded according to an extension of the Clavien-Dindo classification of surgical complications, known as the Japan Clinical Oncology Group postoperative complications criteria. This system describes complications more precisely than the original Clavien-Dindo classification criteria $(15,16)$.

Operative procedure and follow-up. For each patient, the operative procedure was determined based on an extensive, preoperative evaluation of the primary tumor location and the extent of invasion into adjacent organs. When an invasion was detected, resection of the primary tumor was accompanied by a removal of the involved adjacent organ. A lymphadenectomy was not performed, unless obvious findings of lymph node metastasis were observed in the preoperative radiological examination and confirmed during the operation. For each organ removed, both cut ends were examined by preparing frozen sections for histological analysis. Furthermore, microscopic examinations confirmed the final diagnosis of the surgical margin and the presence of invasion into extrahepatic adjacent organs.

After discharge from the hospital, patients had been followed at least for 5 years. Follow-up included a radiological examination with CT or MRI every 6 months and a check for blood test every 3 months. After the liver resection, up to 2003, selected patients received adjuvant treatment with transcatheter hepatic infusion chemotherapy. After 2003, systemic chemotherapy was provided in a clinical trial setting.

Statistics. All data are expressed as the mean \pm standard deviation. Statistical differences between groups were analyzed with the Mann-Whitney U test (continuous variables) or the chi-square test (categorical variables). Disease-free survival and overall survival curves were estimated with the Kaplan-Meier method and analyzed with the log rank test. All statistical analyses were conducted with JMP@11 (SAS Institute Inc., Cary, NC, USA). P-values $<0.05$ were considered as statistically significant. This retrospective study protocol was approved by the institutional reviewer board of the Osaka University Graduate School of Medicine. (No. 15145)

\section{Results}

One hundred seventy-eight patients received liver resections. There were 89 synchronous metastases and 89 metachronous metastases. Ninety-four patients had a single metastasis and 84 patients had multiple metastases. For the synchronous metastases, 53 patients were treated with resections of both the primary colorectal cancer and the metastatic liver tumor in one operation; the other 36 patients were treated with a resection of the primary lesion in first operation and resection of the metastatic lesion in a second operation. There are eight conversion cases from the unresectable liver metastasis to the resectable cases; two cases in the extended resection group and six cases in the non-extended resection group. Table I showed the characteristics of all 178 patients in the extended and the non-extended resection groups. The groups were well-matched in terms of sex, age, body weight, Child-Pugh score, tumor 
Table I. Patient characteristics in liver metastasis.

\begin{tabular}{|c|c|c|c|c|}
\hline Variables & Types & Non-extended $(\mathrm{n}=158)$ & Extended $(n=20)$ & P-value \\
\hline \multirow[t]{2}{*}{ Sex } & Male & 101 & 12 & \multirow[t]{2}{*}{0.8067} \\
\hline & Female & 57 & 8 & \\
\hline Age (years) & & $63.6 \pm 0.8$ & $63.5 \pm 2.4$ & 0.9590 \\
\hline Body weight (kg) & & $58.5 \pm 0.8$ & $58.8 \pm 2.6$ & 0.9076 \\
\hline \multirow[t]{2}{*}{ Number of resections } & First & 156 & 15 & \multirow[t]{2}{*}{0.0002} \\
\hline & Repeated & 2 & 5 & \\
\hline \multirow[t]{2}{*}{ Timing of metastasis } & Synchronous & 84 & 5 & \multirow[t]{2}{*}{0.0306} \\
\hline & Metachronous & 74 & 15 & \\
\hline \multirow[t]{2}{*}{ Tumor number } & Single & 82 & 12 & \multirow[t]{2}{*}{0.6357} \\
\hline & Multiple & 76 & 8 & \\
\hline \multirow[t]{2}{*}{ Neoadjuvant therapy } & Yes & 55 & 9 & \multirow[t]{2}{*}{0.4591} \\
\hline & No & 103 & 11 & \\
\hline \multirow{2}{*}{ Distribution in hepatic lobes } & Unilobar & 109 & 13 & \multirow{2}{*}{0.7992} \\
\hline & Bilobar & 49 & 7 & \\
\hline \multirow[t]{3}{*}{ Child-Pugh score } & A & 156 & 19 & \multirow[t]{3}{*}{0.3021} \\
\hline & B & 2 & 1 & \\
\hline & $\mathrm{C}$ & 0 & 0 & \\
\hline Tumor size (mm) & & $33.0 \pm 1.8$ & $40.9 \pm 5.2$ & 0.1632 \\
\hline \multirow[t]{5}{*}{ Stage (primary tumor) } & I & 5 & 3 & \multirow[t]{5}{*}{0.0762} \\
\hline & II & 30 & 5 & \\
\hline & III & 29 & 5 & \\
\hline & IV & 88 & 5 & \\
\hline & Unknown & 6 & 2 & \\
\hline \multirow[t]{3}{*}{ Adjuvant chemotherapy } & Yes & 103 & 14 & \multirow[t]{3}{*}{0.7463} \\
\hline & No & 55 & 4 & \\
\hline & Unknown & 0 & 2 & \\
\hline \multirow{2}{*}{$\begin{array}{l}\text { Bevacizumab in } \\
\text { perioperative therapy }\end{array}$} & Yes & 16 & 2 & \multirow[t]{2}{*}{1.0000} \\
\hline & No & 142 & 18 & \\
\hline \multirow{3}{*}{$\begin{array}{l}\text { Cetuximab in } \\
\text { perioperative therapy }\end{array}$} & & & & \multirow{3}{*}{0.0977} \\
\hline & Yes & 3 & 2 & \\
\hline & No & 155 & 18 & \\
\hline \multirow{3}{*}{$\begin{array}{l}\text { Panitumumab in } \\
\text { perioperative therapy }\end{array}$} & & & & \multirow{3}{*}{0.0977} \\
\hline & Yes & 3 & 2 & \\
\hline & No & 155 & 18 & \\
\hline \multirow[t]{2}{*}{ Conversion case } & Yes & 6 & 2 & \multirow[t]{2}{*}{0.2224} \\
\hline & No & 152 & 18 & \\
\hline
\end{tabular}

size, and stage of colorectal carcinoma. There is a significant difference in the proportion of synchronous or metachronous metastases $(\mathrm{P}=0.0306)$. As to the number of resections, patients who received repeated liver resections were more frequently observed in the extended resection group than in the non-extended resection group $(\mathrm{P}=0.0002)$. The two groups showed no significant differences in the proportions of single or multiple tumors, unilobar or bilobar distributions in hepatic lobes, the presence of perioperative chemotherapy ,the uase of molecular targeted drugs (bevacizumab, cetuximab and panitumumab) in perioperative chemotherapy. There are eight conversion cases from the unresectable liver metastasis to the resectable cases; two cases in the extended resection group and six cases in the non-extended resection group, although there was no significant difference between the extended group and non-extended group $(\mathrm{P}=0.2224)$.

Table II showed the surgical outcomes of liver resections in the two groups. The mean duration of the operation was significantly longer in the extended resection group (414 min) than in the non-extended resection group ( $308 \mathrm{~min} ; \mathrm{P}=0.0103$ ). The volume of blood loss in the extended resection group was significantly greater than that in the non-extended group $(\mathrm{P}=0.0272)$, but the increased blood loss did not influence the postoperative course or the length of the postoperative hospital 
Table II. Surgical outcomes of liver resections.

\begin{tabular}{|c|c|c|c|c|}
\hline Variables & Types & Non-extended $(n=158)$ & Extended $(n=20)$ & P-value \\
\hline Operation time $(\min )$ & & $307.8 \pm 13.0$ & $414.1 \pm 36.0$ & 0.0103 \\
\hline Blood loss (ml) & & $642.2 \pm 81.8$ & $2025.0 \pm 573.8$ & 0.0272 \\
\hline Blood infusion (ml) & & $127 \pm 418$ & $826 \pm 1528$ & 0.0100 \\
\hline Resected liver weight (g) & & $159.4 \pm 17.1$ & $211.2 \pm 39.2$ & 0.2385 \\
\hline \multirow[t]{2}{*}{ Postoperative complication } & Yes & 24 & 2 & 1.0000 \\
\hline & No & 134 & 18 & \\
\hline \multirow[t]{4}{*}{ Histology } & tub1/2 & 143 & 19 & 0.6113 \\
\hline & por & 4 & 0 & \\
\hline & others & 7 & 0 & \\
\hline & Unknown & 4 & 1 & \\
\hline Postoperative hospital stay (days) & & $24.1 \pm 1.4$ & $23.8 \pm 2.5$ & 0.9247 \\
\hline
\end{tabular}

por, poorly differentiated adenocarcinoma; tub1, well differentiated type tubular adenocarcinoma; tub2, moderately differentiated type tubular adenocarcinoma.

stay, which were similar between the groups. Moreover, the resected liver weight was not significantly different between groups. In both groups, most patients received an R0 resection; $\mathrm{R} 0$ resection rates were $90 \%$ in the extended resection group and $95 \%$ in the non-extended resection group. Among all patients, there were nine $\mathrm{R} 2$ resection cases due to lung metastasis. Postoperative complications were observed in 26 patients, including 24 patients $(15.2 \%)$ in the non-extended resection group and 2 patients $(10.0 \%)$ in the extended resection group. No mortality case occurred in either group.

Concerning about complications, there were 23 patients with postoperative complications. Of the two patients in the extended resection group with postoperative complications, one had wound infection (grade IIIa) and one had bile leakage (grade IIIa). In the non-extended resection group, half the complications $(n=12)$ were wound infections $(n=7$ grade $I, n=1$ grade II, and $n=4$ grade IIIa). Three patients had bile leakage (grades I, II, and IIIa). Four patients had a paralytic ileus after the hepatic resection (grade I, grade II, and 2 grade IIIa); one patient with a grade IIIb intestinal obstruction required a re-operation.Two patients had intra-abdominal abscesses (grades II and IIIa). Two patients had ascites (grade II). One patient with a grade II pulmonary embolism required anticoagulant therapy with warfarin. One with a grade II urinary tract infection and one with drug-induced erythema multiform. Three patients developed two complications; thus, these patients were counted twice.

The organs resected in the extended resection group was shown in Table III, and ten patients had metastatic lesions that invaded the diaphragm, and they received the extended resection of the diaphragm and with a direct closure; no patients required an artificial patch to repair the diaphragm. Five patients received combined resections of IVC. Of these, three patients received continuous suture repairs, and two patients needed reconstructions with ringed polytetrafluoroethylene (PTFE) tube grafts. Three patients received combined resections that included the bile duct. One patient received a direct duct closure, and did not require an anastomosis. Another
Table III. Organs of extended resection.

\begin{tabular}{lr} 
Organs & $\mathrm{n}$ \\
\hline Diaphragm & 10 \\
Inferior vena cava & 5 \\
Bile duct & 3 \\
Hepatic vein & 1 \\
Adrenal gland & 1 \\
Small intestine & 1 \\
Pericardium & 1 \\
Abdominal wall & 1 \\
\hline
\end{tabular}

patient received a bile duct reconstruction with an end to end anastomosis of each bile duct. In two patients, the bile duct resections were combined with a hepaticojejunostomy and a Roux-en-Y anastomosis. Other organs resected included the right hepatic vein, adrenal gland, small intestine, pericardium, and abdominal wall. Three patients received resections of multiple adjacent organs; thus, these patients were counted multiple times.

In the non-extended resection group, 149 patients received $\mathrm{R} 0$ resections, but 86 had tumor recurrences. Tumor relapses were observed in the remnant liver $(n=23)$, in an extra-hepatic organ $(n=30)$, or in both $(n=30)$. In the extra-hepatic organs, most frequent organ was lung $(n=33)$ and secondly lymph nodes. In the extended resection group, 12 patients had tumor recurrences after $\mathrm{R} 0$ resections. Of these, 7 affected the remnant liver, 3 affected extra-hepatic organs, and 2 affected both. Lung metastasis was frequently observed in the extended resection group $(n=3)$. Recurrences at the surgical margin were found in 3 patients in the non-extended resection group, and only one patient in extended resection group. There was no significant difference about local recurrence between two groups $(\mathrm{P}=0.2170)$. About treatments for the recurrence after hepatectomy, surgical resections were performed in 5 cases 

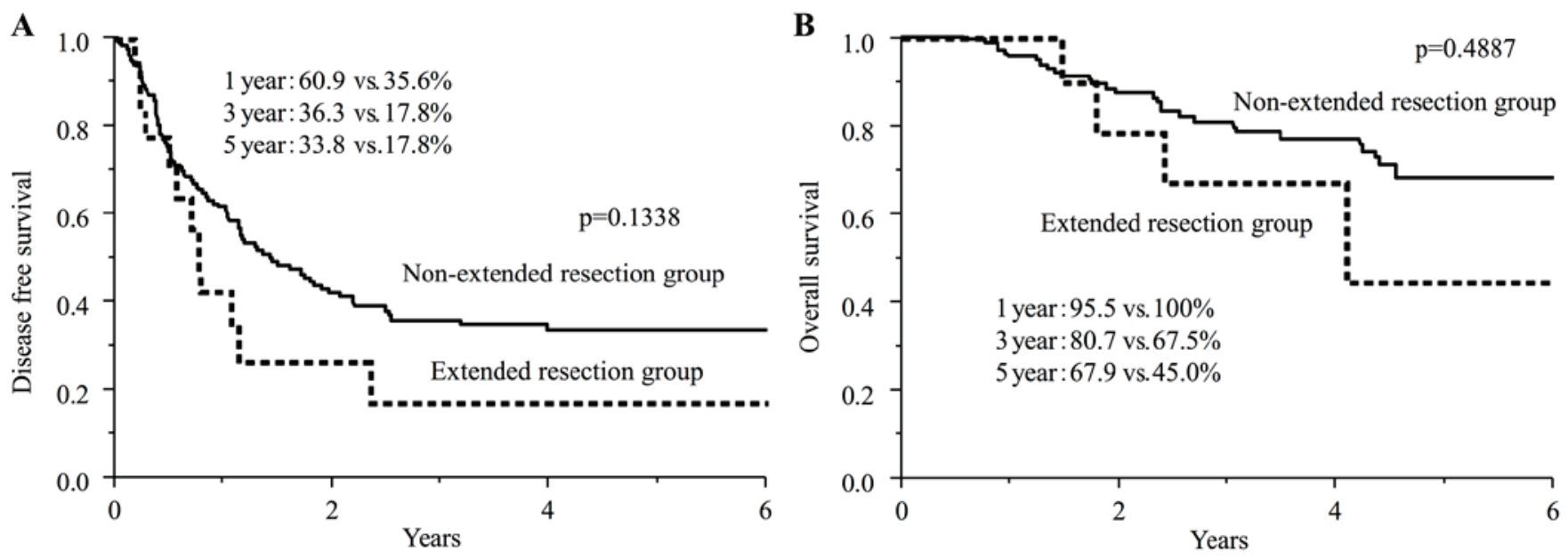

Figure 1. (A) Disease-free survival curves for patients that received non-extended resections and extended resections for treating liver metastases of colorectal cancer. The 1-year, 3-year, and 5-year disease-free survival rates were 35.6, 17.8 and $17.8 \%$ in the extended resection group, and 60.9, 36.3 and 33.8\% in the non-extended resection group. (B) The overall survival curves for patients that received non-extended resections and extended resections. The 1-year, 3-year, and 5-year overall survival rates were $95.5,80.7$ and $67.9 \%$ in the non-extended resection group, and $100,67.5$ and $45.0 \%$ in the extended resection group.

A

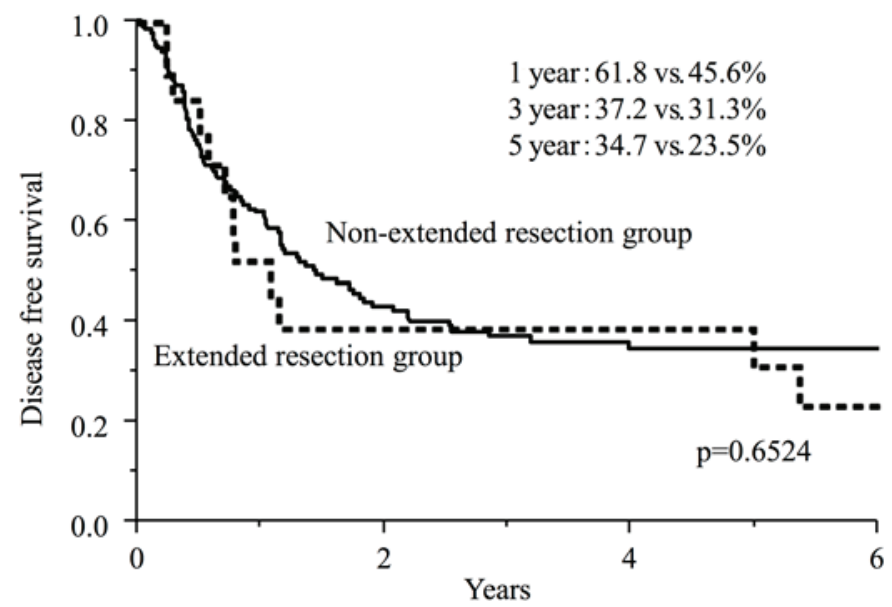

B

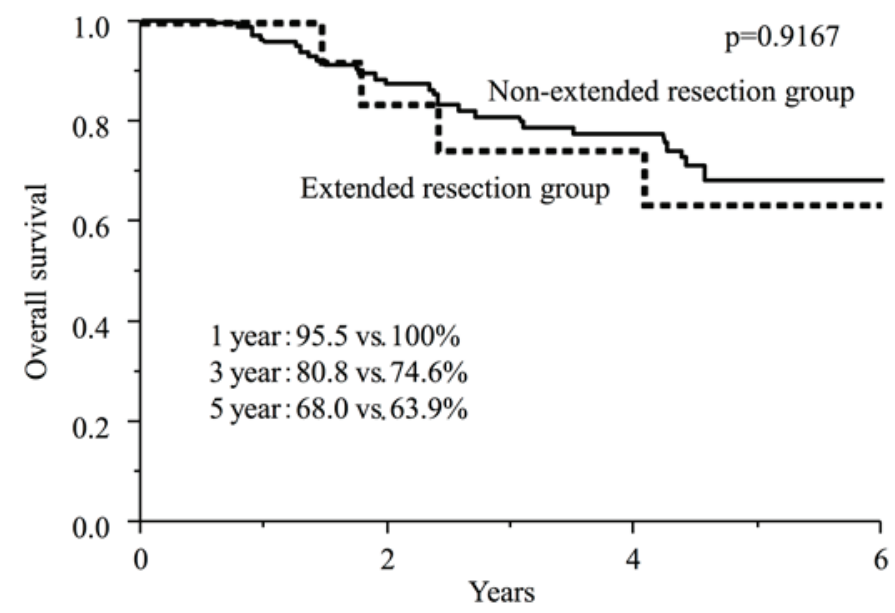

Figure 2. the survival periods from the first hepatectomy. (A) Disease-free survival curves from the first hepatectomy in two groups. The 1-year, 3 -year, and 5-year disease-free survival rates were 45.6,31.3 and 23.5\% in the extended resection group, and 61.8\%, 37.2\%, and 34.7\% in the non-extended resection group. (B) The overall survival curves from the first hepatectomy. The 1-year, 3-year, and 5-year overall survival rates were $95.5,80.8$ and $68.0 \%$ in the non-extended resection group, and 100, 74.6 and $63.9 \%$ in the extended resection group.

in extended resection group and 33 patients in non-extended resection group. A total of 4 patients in extended resection group and 49 patients in non-extended resection group were treated with systemic chemotherapy. Three patients in extended resection group and 4 patients in non-resection group were supported by best supportive care. We could not follow 2 patients in non-extended resection group about treatment after recurrence. There was no significant difference in the treatment for the recurrence after hepatectomy in two groups $(\mathrm{P}=0.2046)$.

Fig. 1 showed the disease-free survival curves and overall survival curves for both groups. The median disease-free survivals were 1.5 years in the non-extended resection group and 0.8 years in the extended resection group. The $1-$ and 3 -year disease-free survival rates were 60.9 and $36.3 \%$ in the non-extended resection group, respectively, and 35.6 and
$17.8 \%$ in the extended resection group, respectively. Although the difference of rates was not significant $(\mathrm{P}=0.1338)$, the disease-free survival rates in the extended resection group tended to be lower than in the non-extended resection group. The 3- and 5-year overall survival rates were 80.7 and $67.9 \%$ in the non-extended resection group, respectively, and $67.5 \%$ and $45.0 \%$ in the extended resection group, respectively. The overall survival rates in extended resection group were not inferior to those in the non-extended resection group,and the difference between two groups was not significant $(\mathrm{P}=0.4887)$.

In the extended resection group, the patients with the repeated resection were significantly frequent as shown in Table I. Fig. 2 showed the disease-free survival curves and overall survival curves of both groups from the timing of first hepatectomy. The 1-year, 3-year, and 5-year disease-free survival rates for the extended resection group were 
45.6, 31.3 and $23.5 \%$, and for the non-extended resection group were $61.8,37.2$ and $34.7 \%$, respectively. These disease survival rates were not significant $(\mathrm{P}=0.6524)$. The 1-year, 3-year, and 5-year overall survival rates were $95.5,80.8$ and $68.0 \%$ in the non-extended resection group, and $100,74.6$ and $63.9 \%$ in the extended resection group. These survival rates were not significant $(\mathrm{P}=0.9167)$.

\section{Discussion}

When colorectal liver metastasis involves an adjacent organ, a hepatectomy combined with resection of the involved adjacent organ is required for macroscopic curative resection. It is important to obtain negative surgical margins, because a positive margin after resection of hepatic colorectal metastases was reported to be associated with increased risk of local recurrence (17). In this study, we analyzed the characteristics and surgical outcomes of patients with colorectal liver metastasis that received a hepatectomy combined with the resection of involved adjacent organs. Among the resected adjacent organs, half involved the diaphragm and one fourth involved IVC.

Previous studies focused on the diaphragm and IVC when evaluating perioperative morbidity and mortality associated with a hepatectomy combined with resection of adjacent organs. Around 2010, several authors reported that liver resections combined with a simultaneous diaphragm excision resulted in high morbidity (44\%) but relative low mortality (3-7\%) compared to a liver resection alone for advanced colorectal liver metastases $(8,9)$. In contrast, conscerning about hepatectomy combined with a IVC resection, several studies reported high morbidity and mortality (40-43 and $8-11 \%$ respectively) $(11,18,19)$. Those findings suggested that a hepatectomy combined with a diaphragm resection required additional attention to postoperative complications, but it could be safely conducted currently. In contrast, a hepatectomy combined with IVC resection remains a challenging procedure due to the high risk of mortality. In our study, the perioperative morbidity was low $(10 \%)$ in the extended resection group, and no mortality cases were observed. The recent advances in surgical techniques and perioperative management might explain the low perioperative morbidity and no mortality case in the present study.

Previous studies reported that patients with liver metastases who received a curative resection had a 5-year survival rate of $35-58 \%$ (6,7). On the other hand, for patients with unresectable liver metastases, the median overall survival was 18-36 months with systemic chemotherapies or hepatic arterial infusion chemotherapy plus systemic chemotherapy $(20,21)$. Thus, hepatectomy is the first recommendation for patients with colorectal liver metastasis lesions. Only a few studies have reported on long-term outcomes for a hepatectomy combined with either a diaphragm excision or IVC resection. Lordan et al reported that a liver resection with a simultaneous diaphragm resection had a worse long-term outcome than a liver resection alone (5-year overall survival rate, 19.6 vs. 62\%; 3-year diseasefree survival rate, 22.1 vs. $50.7 \%$, respectively (8). However, those authors stated that the prognosis for an extended resection was superior to chemotherapy alone. Similarly, in 2012, Li et al reported that patients that received a hepatectomy combined with diaphragm resections for treating colorectal liver metastasis had unfavorable survival rates compared to those with no diaphragm resection (9). About hepatectomy combined with resection of IVC, Miyazaki et al reported a survival rate of $33 \%$ at 3 years after aggressive surgery for colorectal liver metastasis involving IVC in 1999. They concluded that aggressive surgical approaches might provide a favorable outcome in selected patients (22). In 2004, Aoki et al reported that, among patients with metastatic liver tumors from colorectal cancer, patients that received IVC resection or a hepatic venous confluence reconstruction had shorter survival rates than patients without IVC reconstructions (23). From these reports, patients who received a hepatectomy combined with a diaphragm excision or IVC resection had long-term outcomes that were inferior to those of patients that received a hepatectomy alone. In this study, we demonstrated that the survival curves in two groups were overlapped and the differences were not significant. And the 3-year and 5 -year survival rates in the extended resection group in our cohort were $68 \%$ and $45 \%$, and the rates were suprerior to the previous reports.

A total of 8 of 20 patients $(40 \%)$ in our series showed pathological invasion of an adjacent organ in this study. Currently, most institutions conduct a preoperative examination with CT and MRI, and these imaging modalities have been improved; nevertheless, it remains difficult to determine whether a hepatic tumor has invaded an adjacent organ or not. Previously, the rate of proven pathological invasions into adjacent organs associated with colorectal liver metastasis has ranged from 15 to $44 \%(8,24)$, and the rate of confirmed pathological invasions in this study was within that range. Previous studies have identified several indicators for supporting a preoperative diagnosis of invasion into an adjacent organs (24-26). Despite recent radiological advances, it remains difficult to evaluate whether a hepatic tumor has invaded an adjacent organ. We consider that it is necessary to perform a hepatectomy combined with resection of the involved adjacent organ to achieve negative surgical margins for colorectal liver metstasis when there is a strong suspicion that a hepatic tumor has invaded an adjacent organ. Additionally, evaluating the prognosis between the pathologically invasion-positive proup and the invasionnegative group, there was no significant difference in overall survival and disease-free survival rates in this study. When $\mathrm{R} 0$ resection was achieved, it was supposed that the presence of pathological invasion to adjacent organ of colorectal liver metastasis might not influence to the prognosis after hepatectomy.

In this study, the disease-free survival rates and overall survival rates from hepatectomy in extended resection group was inferior to these in non-extended resection group. However, the patients in extended resection group received the repeart hepatectomy more frequently compared with those in non-extended resection group ( 25 vs. $1.3 \%, \mathrm{P}=0.0002)$. This high rate of the repeated resection might influence the diseasefree survival and overall survival rate in extended resection group. With analysis of the survival periods from the first hepatectomy in both groups, there was no difference in both the disease-free survival and the overall survival rate between two groups as shown in Fig. 2.

There is some limitations in this study. Main limitation was that it was conducted in only one institution, and the extended 
group comprised a small number of patients. A larger number of patients might be needed to draw firm conclusions about the long-term outcomes of hepatectomy combined with a resection of adjacent organs for treating colorectal liver metastasis.

In conclusion, we showed that the surgical outcome of a hepatectomy combined with adjacent organ resection was acceptable with low perioperative morbidity although this aggressive operation increased the operation time and the blood loss compared to a hepatectomy alone. We found that the overall survival of the hepatectomy combined with an adjacent organ resection might not be inferior to that of a hepatectomy alone.

\section{Acknowledgements}

Not applicable.

\section{Funding}

No funding was received.

\section{Availability of data and materials}

The datasets used and/or analyzed during the current study are available from the corresponding author on reasonable request.

\section{Authors' contributions}

GS made substantial contributions to acquisition of data and drafting the manuscript. TN and HE made substantial contributions to study conception and design, and revising of the manuscript. HE, YI, DY, TA, KK and KG made contributions to acquisition of data and revising manuscript. SK, YT and MT made substantial contributions to the analysis and interpretation of data. TM, KU, YD and MM revised the paper it critically for important intellectual content, and approved the final version. All authors read and approved the final manuscript.

\section{Ethics approval and consent to participate}

Written informed consent was obtained from all participants. This retrospective study protocol was approved by the Institutional Review Board of the Osaka University Graduate School of Medicine (Suita, Japan). (No. 15145)

\section{Consent for publication}

Not applicable.

\section{Competing interests}

The authors declare that they have no competing interests.

\section{References}

1. Stangl R, Altendorf-Hofmann A, Charnley RM and Scheele J: Factors influencing the natural history of colorectal liver metastases. Lancet 343: 1405-1410, 1994.

2. Petrelli NJ, Abbruzzese J, Mansfield P and Minsky B: Hepatic resection: The last surgical frontier for colorectal cancer. J Clin Oncol 23: 4475-4477, 2005.
3. Watanabe T, Itabashi M, Shimada Y, Tanaka S, Ito Y, Ajioka Y, Hamaguchi T, Hyodo I, Igarashi M, Ishida H, et al; Japanese Society for Cancer of the Colon and Rectum: Japanese Society for Cancer of the Colon and Rectum (JSCCR) Guidelines 2014 for treatment of colorectal cancer. Int J Clin Oncol 20: 207-239, 2015.

4. Stättner S, Primavesi F, Yip VS, Jones RP, Öfner D, Malik HZ, Fenwick SW and Poston GJ: Evolution of surgical microwave ablation for the treatment of colorectal cancer liver metastasis: Review of the literature and a single centre experience. Surg Today 45: 407-415, 2015.

5. Bismuth H, Adam R, Levi F, Farabos C, Waechter F, Castaing D, Majno P and Engerran L: Resection of nonresectable liver metastases from colorectal cancer after neoadjuvant chemotherapy. Ann Surg 224: 509-522, 1996.

6. Weiss L, Grundmann E, Torhorst J, Hartveit F, Moberg I, Eder M, Fenoglio-Preiser CM, Napier J, Horne CH, Lopez MJ, et al: Haematogenous metastatic patterns in colonic carcinoma: An analysis of 1541 necropsies. J Pathol 150: 195-203, 1986.

7. Kopetz S, Chang GJ, Overman MJ,Eng C, Sargent DJ, Larson DW, Grothey A, Vauthey JN, Nagorney DM and McWilliams RR: Improved survival in metastatic colorectal cancer is associated with adoption of hepatic resection and improved chemotherapy. J Clin Oncol 27: 3677-3683, 2009.

8. Lordan JT, Riga A, Worthington TR and Karanjia ND: Early and long-term outcomes of patients undergoing liver resection and diaphragm excision for advanced colorectal liver metastases. Ann R Coll Surg Engl 91: 483-488, 2009.

9. Li GZ, Turley RS, Lidsky ME, Barbas AS, Reddy SK and Clary BM: Impact of simultaneous diaphragm resection during hepatectomy for treatment of metastatic colorectal cancer. J Gastrointest Surg 16: 1508-1515, 2012.

10. Delis SG, Madariaga J and Ciancio G: Combined liver and inferior vena cava resection for hepatic malignancy. J Surg Oncol 96: 258-264, 2007.

11. Malde DJ, Khan A, Prasad KR, Toogood GJ and Lodge JP: Inferior vena cava resection with hepatectomy: Challenging but justified. HPB 13: 802-810, 2011.

12. Colvin H, Mizushima T, Eguchi H, Takiguchi S, Doki Y and Mori M: Gastroenterological surgery in Japan: The past, the present and the future. Ann Gastroenterological Surg 1: 5-10, 2017.

13. Wakayama K, Kamiyama T, Yokoo H, Kakisaka T, Orimo T, Shimada S, Tsuruga Y, Kamachi H and Taketomi A: Our technique of preceding diaphragm resection and partial mobilization of the hepatic right lobe using a vessel sealing device (LigaSure $^{\mathrm{TM}}$ ) for huge hepatic tumors with diaphragm invasion. Surg Today 46: 1224-1229, 2016.

14. Nelson H, Petrelli N, Carlin A, Couture J, Fleshman J, Guillem J, Miedema B, Ota D and Sargent D; National Cancer Institute Expert Panel: Guidelines 2000 for colon and rectal cancer surgery. J Natl Cancer Inst 93: 583-596, 2001.

15. Dindo D, Demartines N and Clavien P-A: Classification of surgical complications: A new proposal with evaluation in a cohort of 6336 patients and results of a survey. Ann Surg 240: 205-213, 2004.

16. Katayama H, Kurokawa Y, Nakamura K, Ito H, Kanemitsu Y, Masuda N, Tsubosa Y, Satoh T, Yokomizo A, Fukuda H, et al: Extended Clavien-Dindo classification of surgical complications: Japan Clinical Oncology Group postoperative complications criteria. Surg Today 46: 668-685, 2016.

17. Pawlik TM, Scoggins CR, Zorzi D, Abdalla EK, Andres A, Eng C, Curley SA, Loyer EM, Muratore A, Mentha G, Capussotti L and Vauthey JN: Effect of surgical margin status on survival and site of recurrence after hepatic resection for colorectal metastases. Ann Surg 241: 715-722, 2005.

18. Arii S, Teramoto K, Kawamura T, Takamatsu S, Sato E, Nakamura N, Iwai T, Mori A, Tanaka J and Imamura M: Significance of hepatic resection combined with inferior vena cava resection and its reconstruction with expanded polytetrafluoroethylene for treatment of liver tumors. J Am Coll Surg 196: 243-249, 2003.

19. Hemming AW, Mekeel KL, Zendejas I, Kim RD, Sicklick JK and Reed AI: Resection of the liver and inferior vena cava for hepatic malignancy. J Am Coll Surg 217: 115-124; discussion 124-115, 2013.

20. Kemeny N, Jarnagin W, Paty P, Gönen M, Schwartz L, Morse M, Leonard G, D'Angelica M, DeMatteo R, Blumgart L, et al: Phase I trial of systemic oxaliplatin combination chemotherapy with hepatic arterial infusion in patients with unresectable liver metastases from colorectal cancer. J Clin Oncol 23: 4888-4896, 2005. 
21. Cascinu S, Berardi R, Salvagni S, Beretta GD, Catalano V, Pucci F, Sobrero A, Tagliaferri P, Labianca R, Scartozzi M, et al: A combination of gefitinib and FOLFOX-4 as first-line treatment in advanced colorectal cancer patients. A GISCAD multicentre phase II study including a biological analysis of EGFR overexpression, amplification and NF-kB activation. Br J Cancer 98: 71-76, 2008.

22. Miyazaki M, Ito H, Nakagawa K, Ambiru S, Shimizu H, Okuno A, Nukui Y, Yoshitomi H, Kusashio K, Furuya S, et al: Aggressive surgical resection for hepatic metastases involving the inferior vena cava. Am J Surg 177: 294-298, 1999.

23. Aoki T, Sugawara Y, Imamura H, Seyama Y, Minagawa M, Hasegawa K, Kokudo N and Makuuchi M: Hepatic resection with reconstruction of the inferior vena cava or hepatic venous confluence for metastatic liver tumor from colorectal cancer. J Am Coll Surg 198: 366-372, 2004.
24. Hashimoto T, Minagawa M, Aoki T, Hasegawa K, Sano K, Imamura H, Sugawara Y, Makuuchi M and Kokudo N: Caval invasion by liver tumor is limited. J Am Coll Surg 207: 383-392, 2008.

25. Lau WY, Leung KL, Leung TW, Liew CT, Chan M and Li AK Resection of hepatocellular carcinoma with diaphragmatic invasion. Br J Surg 82: 264-266, 1995.

26. Yamashita Y, Morita K, Iguchi T, Tsujita E, Soejima Y, Taketomi A and Maehara Y: Surgical impacts of an en bloc resection of the diaphragm for hepatocellular carcinoma with gross diaphragmatic involvement. Surg Today 41: 101-106, 2011. 